\title{
Pseudotumour presentation of pulmonary tuberculosis
}

\author{
Jane S. Afriyie-Mensah ${ }^{1}$, Felix R. Awindaogo ${ }^{2}$, Samuel Kofi Asomani ${ }^{2}$
}

Ghana Med J 2020; 54(2): 126-130 DOI: http://dx.doi.org/10.4314/gmj.v54i2.12

\author{
${ }^{1}$ University of Ghana Medical School, Department of Medicine and Therapeutics, Accra, Greater Accra, \\ Ghana \\ ${ }^{2}$ Korle Bu Teaching Hospital, Department of Medicine and Therapeutics, Accra, Greater Accra, Ghana
}

Corresponding author: Jane S. Afriyie-Mensah

Conflict of interest: None declared

E-mail: jafriyiemensah@yahoo.com

\begin{abstract}
SUMMARY
Pulmonary tuberculosis manifesting as a mass lesion, thus, mimicking a lung carcinoma is an unusual radiographic presentation of tuberculosis (TB). The common radiologic patterns and clinical presentations are well known and documented. We report two cases of pulmonary tuberculosis with a neoplastic appearance on chest imaging diagnosed histologically. A 21 - year old female with cough, weight loss, anorexia and an unremarkable physical examination. Chest radiography showed a right apical mass suggestive of lung cancer. Histology of the lesion revealed parenchymal pulmonary tuberculosis. A 49-year old male with left-sided chest pain, cough, anorexia, weight loss, mild pallor with an unremarkable chest examination. Chest imaging showed a left apical mass and mediastinal lymphadenopathy. Microscopic examination of the mass confirmed pulmonary tuberculosis. Pseudotumour pulmonary tuberculosis is a rare clinical entity that can lead to diagnostic challenges and must be considered in the differential diagnosis when mass lesions are seen on chest imaging, especially in TB endemic areas.
\end{abstract}

Keywords: tuberculosis, pulmonary, carcinoma, atypical, diagnostic

Funding: None declared

\section{INTRODUCTION}

Pulmonary tuberculosis (PTB) accounts for approximately $80 \%$ of all forms of tuberculosis (TB) in adults and characterized by a wide range of radiological and clinical features. ${ }^{1}$ It is the commonest form of tuberculosis in Ghana and constitutes about $92 \%$ of newly diagnosed and relapsed cases. About 44000 new cases of tuberculosis was reported in $2018 .^{2}$

The common radiological manifestations of PTB are well known and documented but can sometimes present with atypical or unusual radiological patterns which results in diagnostic quagmires culminating in delayed diagnosis and treatment. ${ }^{3} \mathrm{~A}$ distinct radiographic manifestation of PTB mimicking a lung carcinoma is pseudotumoural pulmonary tuberculosis. ${ }^{4,5}$ Pseudotumoural pulmonary tuberculosis is rare and occurs in $3.5 \%$ to $4.5 \%$ of immunocompetent patients even in $\mathrm{TB}$ endemic regions of the world. ${ }^{6,7}$

Diagnosis is usually challenging because of non-specific clinical features and radiological similarities to lung cancer and other lung pathologies. ${ }^{8,9}$ Short course anti-tuberculous treatment as for other forms of pulmonary tuberculosis consisting of isoniazid (INH), rifampicin (RIF), ethambutol (EMB) and pyrazinamide (MPZ) has been found to be effective in controlling the disease and reducing the size of the mass. ${ }^{10,11} \mathrm{We}$ present two cases of pulmonary tuberculosis mimicking lung carcinoma.

\section{CASE REPORT 1}

A 21 - year old female patient with complaints of cough productive of scanty whitish sputum, fever, weight loss and anorexia of three months duration. She had unsuccessfully received multiple antibiotic courses for the cough. On physical examination, she looked well, afebrile with evidence of weight loss and had a normal chest examination. The biochemical, immunological and haematological tests were normal (Table 1). The erythrocyte sedimentation rate (ESR) was elevated $(39 \mathrm{~mm} / \mathrm{hr}$ ), however, sputum for acid-fast bacilli (AFB) and GeneXpert (GeneXpert Dx System Version 4.8) did not show the presence of mycobacterium tuberculosis.

A well-defined smooth, homogenous right apical opacity was observed on the posteroanterior (PA) chest $\mathrm{x}$-ray (Figure 1a). Computed tomography scan (CT scan) of the chest revealed a spiculated right upper lobe mass. The mass measured $31.4 \times 35.3 \times 2.75 \mathrm{~mm}$ with anterior pleural attachment and satellite nodular opacifications around it suggesting a bronchogenic carcinoma (Figure 1b). 


\section{Case Report}

There was no associated mediastinal lymphadenopathy. Microscopic examination of lung tissue obtained via CT guided biopsy showed diffuse chronic inflammatory changes with multiple small necrotizing granulomata and interstitial fibrosis consistent with tuberculosis. Standard anti - tuberculosis treatment was initiated and resulted in complete resolution of the lesion as well as her symptoms (Figure 1c).
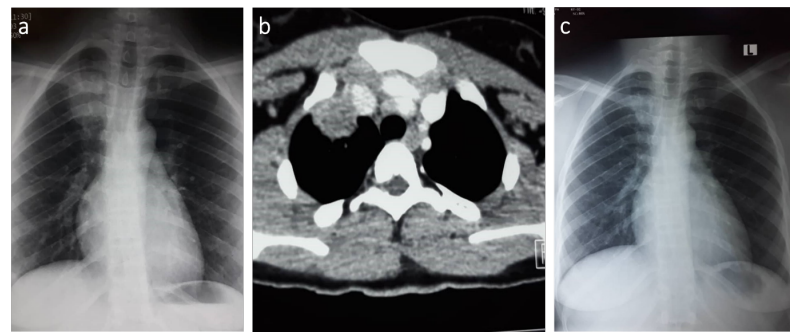

Figure 1 Radiological images for Case 1

a. Chest $\mathrm{x}$ - ray showing a well - defined left apical opacity.

b. Contrast - enhanced CT scan shows an irregular, spiculated anteroapical mass.

c. Chest $\mathrm{x}$ - ray showing resolution of the opacity post treatment.

Table 1 Laboratory results

\begin{tabular}{|c|c|c|}
\hline Laboratory investigation & Case 1 & Case 2 \\
\hline Haemoglobin (g/dL) & $12.0(11.5-16.5)$ & $10.2(13.0-18.0)$ \\
\hline Mean corpuscular volume (fL) & $88(76-99)$ & $86(76-99)$ \\
\hline Mean corpuscular haemoglobin (pg) & $29.8(26-34)$ & $28(26-33)$ \\
\hline Platelets $\left(\times 10^{9} / \mathrm{l}\right)$ & $271(150-450)$ & $330(150-450)$ \\
\hline Total white cell count $\left(\times 10^{9} / 1\right)$ & $5.3(4.0-12.0)$ & $4.3(4.0-12.0)$ \\
\hline ESR mmfall/hr & $39(\leq 20)$ & $(\leq 15)$ \\
\hline Sputum GeneXpert & No MTB & No MTB \\
\hline Bronchial wash GeneXpert & - & No MTB \\
\hline Sputum for AFB & No AFB & No AFB \\
\hline $\begin{array}{l}\text { Serum angiotensin converting en- } \\
\text { zyme }\end{array}$ & $27.8(0-52)$ & - \\
\hline Serum ionised calcium & $1.18(1.06-1.30)$ & - \\
\hline HIV 1 and 2 & Non - reactive & Non - reactive \\
\hline
\end{tabular}

$\mathrm{MTB}=$ Mycobacterium tuberculosis; $\mathrm{AFB}=\mathrm{Acid}-$ fast bacilli

\section{CASE REPORT 2}

A 49-year old male with left-sided chest pain and a productive cough (copious whitish sputum) of three months duration associated with anorexia, lethargy, significant weight loss and an episode of haemoptysis. Chest pain was of moderate severity, non-radiating, pleuritic and relieved temporarily with analgesics. He looked chronically ill and cachexic with mild pallor on physical examination. Chest and other systems examination was unremarkable.

Laboratory tests showed normochromic normocytic anaemia and a normal ESR. Mycobacterium tuberculosis was not detected on examination of sputum (Table 1).

The chest $\mathrm{x}$ - ray showed a well - defined opacification of the upper third of the left lung field abutting the medi- astinum and mildly displacing the distal trachea and carina to the right suggesting a filling defect in the distal trachea (Figure 2a). A mass with irregular margins and a few linear and circular lucencies in the apex of the left lung measuring $70.0 \times 36.5 \mathrm{~mm}$ was noted on CT scan of the chest. The mass abutted the arch of the aorta and descending aorta as well as the main pulmonary trunk (Figure $2 b$ and $2 c)$. Mediastinal lymphadenopathy was also noted. Bronchoalveolar lavage for acid - fast bacilli and GeneXpert were also negative. Microscopic examination of lung tissue obtained via CT guided percutaneous biopsy showed few caseating epithelioid granulomas with some showing central neutrophilic debris and moderate lymphoplasmacytic infiltrates in the interstitium suggestive of pulmonary TB.

He received standard anti - tuberculosis treatment with complete symptom and radiological resolution of lung mass after 5 months of treatment (Figure 2d).

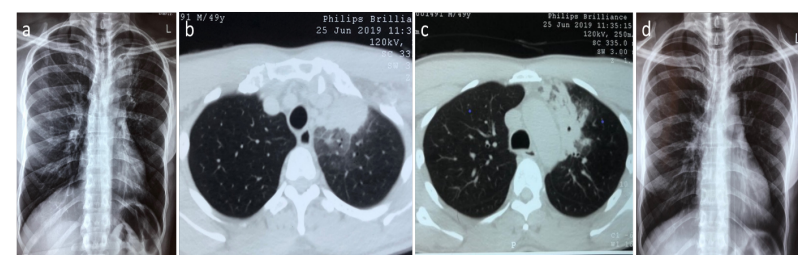

Figure 2. Radiological images for Case 2

a. Chest $\mathrm{x}$-ray showing well - defined left upper-lobe opacity.

b. Contrast - enhanced CT scan showing the mass and enlarged mediastinal lymph nodes.

c. Contrast - enhanced CT scan showing the mass abutting the ascending aorta.

d. Chest $\mathrm{x}$ - ray showing resolution of the mass after 5 months of treatment

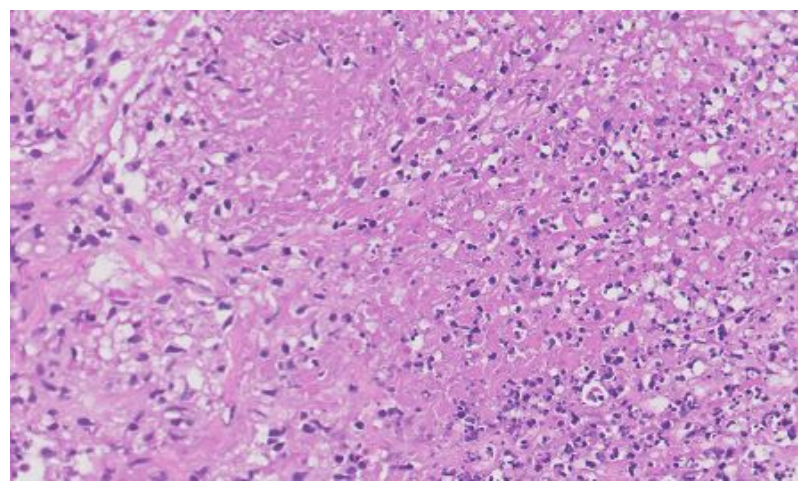

Figure 3. Histology of lesion of Case 2 showing epithelioid granulomas with caseous necrosis and moderate lymphoplasmacytosis

\section{DISCUSSION}




\section{Case Report}

Tuberculosis is currently one of the most important infectious diseases globally and is the leading cause of death from a single infectious agent accountable for an estimated 1.6 million deaths in 2017. ${ }^{12}$ Tuberculosis affects all organs of the body with the lungs being the most frequently affected. ${ }^{1}$ Lung cancer is the most commonly diagnosed cancer and the leading cause of cancer - related deaths globally and is responsible for 1.8 million deaths in 2018. ${ }^{13,14}$

Pulmonary tuberculosis (PTB) and lung cancer may be difficult to distinguish clinically and radiologically. ${ }^{15}$ Clinical symptoms such as weight loss, chest pain, anorexia, productive cough and haemoptysis are common in both conditions and respiratory examination may be unremarkable. ${ }^{16}$ Majority of these symptoms were noted in both cases presented above. The radiological presentation of PTB depends on whether it is primary or post primary TB. Primary PTB, common in children, is usually seen as dense, homogeneous, segmental, lobar, or multifocal unilateral consolidation associated with ipsilateral lymphadenopathy (TB lymphadenitis) on chest $\mathrm{x}-$ ray. ${ }^{17}$ Other findings may include right - sided atelectasis, unilateral pleural effusion and miliary nodules. In contrast, post - primary pulmonary tuberculosis, common in the adult population, may manifest radiologically as patchy, heterogeneous consolidation involving the apical and posterior segments of the upper lobes and superior segments of the lower lobes with ill - defined borders and small satellite nodules. ${ }^{17}$ Cavitations, calcifications, nodular or linear opacities, miliary nodules and unilateral loculated pleural effusion are also common findings in post-primary TB. ${ }^{18}$

The manifestation of PTB as a lung mass is quite rare in immunocompetent individuals and differentiation from a primary lung cancer becomes challenging especially when repeated negative sputum smears for acid - fast bacilli, sputum culture for MTB and GeneXpert occurs. ${ }^{6,11}$ Conversely, lung cancer can also be misdiagnosed as PTB, particularly in the regions of the world where tuberculosis is endemic with a very high burden. ${ }^{16,19}$ Anecdotal evidence in our environment adds credence to this observation as a significant proportion of primary lung cancer cases receive prior TB treatment delaying the diagnosis with abysmal outcomes. In addition, the diagnosis of lung cancer may be unnoticed when both tuberculosis and lung cancer occur concurrently in extremely rare circumstances where sputum examination for the presence of mycobacterium is positive. ${ }^{16,20}$
Lung cancer, on the other hand, is usually seen as a solitary pulmonary nodule or mass greater than $30 \mathrm{~mm}$ and may have ill - defined, spiculated, or well - defined borders with/without associated hilar and/or mediastinal lymphadenopathy. The presence of a pleural tag, amorphous calcification, feeding vessel sign, positive bronchus sign and thick-walled irregular cavities may be other features of lung cancer on chest CT scan. ${ }^{21,22}$

Atypical manifestations of PTB in immunocompetent individuals reported in literature include lymphadenopathy without parenchymal infiltrates, lower lobe disease without lymphadenopathy, solitary tuberculoma or mass density and the occurrence of primary disease in patients older than 40 years. These unusual patterns are largely responsible for misdiagnosis or delayed diagnosis resulting in increased mortality and morbidity. ${ }^{5,6,9}$ It has been estimated that about $3-6 \%$ of pulmonary TB may present as non-calcified mass lesions which are best characterized on chest CT-scan usually measuring between 5 $\mathrm{mm}$ to $40 \mathrm{~mm}$ in the largest diameter. ${ }^{18}$ Similarly the cases presented here were both immunocompetent individuals who presented unusually with lung masses of 35 $\mathrm{mm}$ and $70 \mathrm{~mm}$ in their largest diameter. In the case of the young lady, the size of the mass and the spiculated margins raised the suspicion of a malignant lung mass with differentials of primary pulmonary lymphoma or primary lung cancer although her age, the presence of satellite lesions and the well-defined margins of the lung mass also made inflammatory/infectious causes (PTB, atypical presentation of pulmonary sarcoid) likely. ${ }^{23}$

It is worth noting that a small proportion of benign lung nodules/masses may exhibit spiculated margins whereas about $20 \%$ of primary lung cancers may show well - defined margins. ${ }^{24}$ In the case of the 49 - year old, the bigger mass size, irregular margins, proximity to the pulmonary trunk and aorta as well as the involvement of mediastinal lymph nodes heightened the suspicion of a primary lung cancer. Although rare and non - specific, mediastinal lymphadenopathy may be detected (as in our second case) in about $5 \%$ of post-primary PTB cases. ${ }^{6,25}$ As in our cases, clinical signs are usually absent or nonspecific in parenchymal pseudotumoural PTB and microscopic examination of the sputum as well as molecular tests are often negative for MTB because of the encapsulated nature and poor oxygenation of the solid caseous lesions. ${ }^{11,25}$ Invasive diagnostic techniques and histological examination of the mass usually clinches the diagnosis since traditional methods are usually disappointing amidst the diagnostic dilemma. 
As expected for common forms of PTB, the response to anti-tuberculous regimen in pseudotumoural PTB is phenomenal judging from the post TB treatment chest images of the cases presented. ${ }^{10,26}$

The presentation of PTB as a mass lesion radiographically is uncommon and can be mistaken for a neoplastic formation with the similar clinical presentation compounding the diagnostic dilemma.

\section{CONCLUSION}

Pulmonary tuberculosis should always be a differential diagnosis until proven otherwise when mass lesions are observed on chest imaging regardless of the radiographic features and clinical history, especially in TB endemic areas.

\section{REFERENCES}

1. Thwaites G. Tuberculosis. In: Manson's Tropical Infectious Diseases. Elsevier; 2014:468-505.e3. doi:10.1016/B978-0-7020-5101-2.00041-8

2. World Health Organization. Global Tuberculosis Report 2019. Geneva; 2019.

3. Dalar L, Sokucu SN, Karasulu AL, Altin S. Tuberculosis Can Mimic Lung Cancer: A Case Series. Turkish Thorac Journal/Türk Toraks Derg. 2013;14(1):30-35. doi:10.5152/ttd.2013.06

4. Campobasso A, Perrone A, D'Introno A, Boccuti V, Sangineto M, Resta L, et al. Pulmonary pseudotumoral tuberculosis in an old man: A rare presentation. Case Reports Intern Med. 2017;4(1):78. doi:10.5430/crim.v4n1p78

5. Cherian MJ, Dahniya MH, Al-Marzouk N, Osmanagich E, Abul A, Haider A. Primary pulmonary tuberculosis presenting as mass lesions and simulating tumours in children. Australas Radiol. 1998;42(4):309-312. doi:10.1111/j.14401673.1998.tb00528.x

6. Herrak L, Amangar N, Berri K, El Begnani M, El Ftouh M, El Fassy Fihry MT. Pulmonary tuberculosis in its pseudotumoral form; one new case. Egypt $J$ Chest Dis Tuberc. 2013;62(4):647-649. doi:10.1016/j.ejcdt.2013.08.007

7. Nèji H, Affes M, Attia M, Ben Saad S, Berraies A, Baccouche I, et al. Atypical Pleuropulmonary Tuberculosis Mimicking a Malignant Disease. $J$ Thorac Oncol.2017;12(9):e145-e147. doi:10.1016/j.jtho.2017.05.003

8. Paci M, Cavazza A, Ferrari G, Filice A, Sgarbi G. Pulmonary Tuberculosis Mimicking Lung Metastasis. J Thorac Oncol. 2008;3(6):660-661. doi:10.1097/JTO.0b013e3181757ad4

9. Agarwal R, Srinivas R, Aggarwal AN. Parenchymal pseudotumoral tuberculosis: Case series and systematic review of literature. Respir Med. 2008; 102(3):382-389. doi:10.1016/j.rmed.2007.10.017

10. Lee HS, Oh JY, Lee JH, Yoo CG, Lee C-T, Kim YW, et al. Response of pulmonary tuberculomas to anti-tuberculous treatment. Eur Respir J. 2004; 23(3):452 LP - 455. doi:10.1183/09031936.04.00087304

11. Ishida T, Yokoyama H, Aneko SK, Sugio K, Sugimachi K, Hara N. Pulmonary tuberculoma and indications for surgery: radiographic and clinicopathological analysis. Respir Med. 1992;86(5):431436. doi:10.1016/S0954-6111(06)80011-9

12. World Health Organization, ed. WHO | Global Tuberculosis Report 2018. Geneva; 2018.

13. Bray F, Krug E. New Statistics Show Cancer Burden Rising In The World, Lung Cancer Biggest Killer. Health Policy Watch. 2019:2-5. https://www.healthpolicy-watch.org/new-statisticsshow-cancer-burden-rising-in-the-world-lung-cancer-biggest-killer.

14. The International Agency for Research on Cancer (IARC) report W. Latest global cancer data: Cancer burden rises to 18.1 million new cases and 9.6 million cancer deaths in 2018. Int Agency Res Cancer. 2018;(September):13-15. http://gco.iarc.fr/.

15. Snene H, Ben Mansour A, Toujani S, Ben Salah N, Mjid M, Ouahchi Y et al. Tuberculous pseudotumour, a challenging diagnosis. Rev Mal Respir. 2018;35(3):295-304 doi: 10.1016/j.rmr.2017.03.038

16. Falagas ME, Kouranos VD, Athanassa Z, Kopterides P. Tuberculosis and malignancy. Qjm. 2010;103(7):461-487. doi:10.1093/qjmed/hcq068

17. Leung AN. Pulmonary tuberculosis: The essentials. Radiology. 1999;210(2):307-322. doi:10.1148/radiology.210.2.r99ja34307

18. Curvo-Semedo L, Teixeira L, Caseiro-Alves F. Tuberculosis of the chest. Eur J Radiol. 2005;55(2): 158-172. doi: 10.1016/j.ejrad.2005.04.014

19. Singh VK, Chandra S, Kumar S, Pangtey G, Mohan A, Guleria R. A common medical error: Lung cancer misdiagnosed as sputum negative tuberculosis. Asian Pacific J Cancer Prev. 2009;10(3):335-338.

20. Ammor FZ, Rabiou S, Issoufou I, Belliraj L, Ghalimi J, Serraj M, et al. (2016). Anterior Mediastinal Pseudo- Tumor Tuberculosis: Should We Settle for This Diagnosis? J Case Rep Stud 4(2): 211. doi: 10.15744/2348- 9820.4.211

21. Parker MS, Rosado-de-Christenson ML, Abbott GF. Chest Imaging Case Atlas. Stuttgart: Georg Thieme Verlag; 2012:304-312. doi:10.1055/b0034-98220

22. Sureka B, Thukral B, Mittal M, Mittal A, Sinha M. What clinicians need to know about imaging features in lung cancer? J Mahatma Gandhi Inst Med 


\section{Case Report}

Sci. 2014;19(2):100. doi:10.4103/09719903.138428

23. Truong MT, Sabloff BS, Ko JP. Multidetector CT of Solitary Pulmonary Nodules Thorac Surg Clin. 2010;20(1):9-23.

doi: 10.1016/j.thorsurg.2009.12.002

24. Gumey JW. Determining the likelihood of malignancy in solitary pulmonary nodules with Bayesian analysis. Radiology. 1993;(186):405.
25. Franco R, Santana MA, Coelho Filho JC, PereiraSilva JL. Pseudotumoral form of primary progressive tuberculosis: a diagnosis to be considered. Braz J Infect Dis. 2003;7(2):166-170. doi:10.1590/S1413-86702003000200011

26. Daoudi M, Herrak L, El Ftouh M, Achachi L. Forme pseudo-tumorale d'une tuberculose bronchopulmonaire chez un immunocompétent mimant un cancer. Pan Afr Med J. 2019; 32:170. doi:10.11604/pamj.2019.32.170.1812 